\title{
Driven and damped double sine-Gordon equation: The influence of internal modes on the soliton ratchet mobility
}

\author{
Niurka R. Quintero, ${ }^{1, *}$ R. Alvarez-Nodarse, ${ }^{2, \dagger}$ and Franz G. Mertens ${ }^{3, \ddagger}$ \\ ${ }^{1}$ Departamento de Física Aplicada I, E.U.P., Universidad de Sevilla, c/Virgen de África 7, 41011 Sevilla, Spain \\ ${ }^{2}$ Departamento de Análisis Matemático and IMUS, Universidad de Sevilla, Apdo. 1180, 41080 Sevilla, Spain \\ ${ }^{3}$ Physikalisches Institut, Universität Bayreuth, 95440 Bayreuth, Germany
}

(Received 22 April 2009; published 28 July 2009)

\begin{abstract}
This work studies the damped double sine-Gordon equation driven by a biharmonic force, where a parameter $\lambda$ controls the existence and the frequency of an internal mode. The role of internal oscillations of the kink width in ratchet dynamics of kink is investigated within the framework of collective coordinate theories. It is found that the ratchet velocity of the kink, when an internal mode appears in this system, decreases contrary to what was expected. It is also shown that the kink exhibits a higher mobility in the double sine-Gordon without internal mode, but with a quasilocalized first phonon mode.
\end{abstract}

DOI: 10.1103/PhysRevE.80.016605

PACS number(s): 05.45.Yv, 05.60.Cd, 63.20.Pw

\section{INTRODUCTION}

The ratchet effect in point-particle systems is a well known phenomenon [1] (see also [2-4]), in which a combination of periodic forces/noise and an asymmetric periodic potential allows a particle (or a system of two particles with internal degree of freedom [5]) to break some kind of symmetry so that a finite current may exist even when perturbations acting on the system have zero average.

It is quite common in many phenomena such as scattering and diffusion that solitons, nonlinear coherent excitations in extended systems, also behave like particles. This fact makes it possible to reduce the infinite number of degrees of freedom of extended systems to only one, i.e., the dynamics of soliton may be described in terms of a so-called collective coordinate (CC) [6]. The ratchet effect has been studied also in extended systems, first in the overdamped and underdamped asymmetric sine-Gordon $(\mathrm{sG})$ equation $[7,8]$ and later on in the sG equation with localized and periodic inhomogeneities under the action of an ac force [9]. In all these cases the point-particle description for the soliton captures the ratchet phenomenology (at least qualitatively) by using only one collective variable. The consideration of a soliton as an extended object with a variable width, instead of a rigid particle, is used to explain the ratchet mechanism and also to improve analytical results $[9,10]$. However, to perform experimental realizations corresponding to such ratchet models it is necessary to change the geometry of the Joshepson Junctions (JJ), first in order to induce spatial asymmetry and second, in order to study the fluxon mobility as a function of external parameters.

For this reason, a soliton ratchet has been proposed theoretically [11] and observed experimentally in JJ [12,13] (for other experimental realizations of ratchets in JJ see [14]), when, by using a biharmonic ac force, temporal symmetries are broken instead of spatial ones. This system is modeled by

\footnotetext{
*niurka@us.es

†ran@us.es

†ranz.mertens@uni-bayreuth.de
}

the damped and driven $\mathrm{sG}$ equation and has been extensively analyzed in the framework of collective coordinate approaches $[11,15,16]$. Furthermore, the ratchet dynamics of a kink in the damped and driven $\phi^{4}$ equation was also investigated and compared with the sG case [16]. Both models belong to the perturbed nonlinear Klein-Gordon systems; however the $\phi^{4}$ soliton has one internal mode (IM) related to oscillations of the kink width; whereas in the $\mathrm{sG}$ soliton there are none [17], but a similar role can be played by certain phonon modes [18]. In this regard, by using a collective coordinate approach with two degrees of freedom, namely the center of the soliton, $X(t)$, and its width, $l(t)$, it has been shown that the deformations of the soliton play a crucial role in the mechanism of this soliton ratchet [16] as well in the $\mathrm{sG}$ as in the $\phi^{4}$ model. Indeed, while the average of the external ac force is always zero, a nonzero effective force, modulated by the kink width, acts on the center of the kink and a direct transport can be induced. The amplitude of this effective force is also related with the topological charge and the mass of the $\mathrm{sG}$ or $\phi^{4}$ kinks, and so a $\phi^{4}$ kink exhibits a higher mobility. Therefore, it might be speculated that internal modes in extended systems supporting topological solitons enhance the ratchet mobility of kinks. It is the purpose of this work, however, to show that this is not the case.

In order to address this question we study the ratchet dynamic of a kink in the damped and driven double sineGordon (dsG) equation

$$
\begin{gathered}
\phi_{t t}-\phi_{x x}=-\frac{d U}{d \phi}-\beta \phi_{t}+f(t), \\
U(\phi, \lambda)=(1-\cos \phi)+\frac{\lambda}{2}(1-\cos 2 \phi),
\end{gathered}
$$

with a $T$-periodic force given by

$$
f(t)=\epsilon_{1} \sin \left(\omega t+\theta_{1}\right)+\epsilon_{2} \sin \left(2 \omega t+\theta_{2}\right),
$$

where $\beta$ is the damping coefficient, $\lambda$ is a parameter $(|\lambda|$ $<1 / 2) ; T=2 \pi / \omega$, and $\epsilon_{1}$ and $\epsilon_{2}$ and $\theta_{1}$ and $\theta_{2}$ represent the amplitudes and the phases, respectively, of each harmonic. In this model $\lambda$ controls the shape of the potential $U(\phi, \lambda)$, the 
kink mass and the existence and frequency of an internal mode, which appears only for positive values of $\lambda$. We show that a kink moves slower and slower when we increase $\lambda$ from zero to $1 / 2$. On the other hand, for $\lambda<0$, i.e. in the case of a nonintegrable model without IMs, the kink exhibits higher mobility going from $\lambda=0$ to $-1 / 2$. Eq. (1) serves as a model for low-dimensional magnetic systems (see [19] and references therein). It was also used to describe the defect propagation in hydrogen-bonded molecular chains [20]. Moreover, it is related to one-dimensional arrays of inductively coupled superconducting quantum interference devices (SQUIDs) (see [14], where a single asymmetric element of such an array was considered). Apart from these specific applications, the study of the effects of internal modes is a very important issue since these modes are quite common in nonlinear systems, either intrinsically or as a result of small perturbations [21].

The rest of the paper is organized as follows. In Sec. II we use two collective coordinate methods for finding the equations of motion fulfilled by collective variables and give an approximate expression for the average ratchet velocity. In the next section, by means of simulations we check the predictions of the CC approaches. In Sec. IV the main findings of our work are briefly summarized and their implications for other systems such as the Peyrard-Remoissenet (PR) potential [22] are also discussed. A numerical analysis of the spectrum of linear excitations around the static kink solution in the $\mathrm{dsG}$ equation is provided in an Appendix.

\section{COLLECTIVE COORDINATE APPROACHES}

The aim of this section is to obtain an approximate expression for the average ratchet velocity of the kink center in the dsG when $|\lambda|<1 / 2$. To this end, we use two different CC approaches proposed in [23-26] (see [27] for a review).

\section{A. First ansatz: one collective coordinate.}

It is well known that for $|\lambda|<1 / 2$ the kink solution of the $\mathrm{ds} G$ equation is represented by

$$
\phi_{1}(x, t)= \begin{cases}-2 \arctan \Xi(x, t), & x-X(t) \leq 0, \\ 2 \pi-2 \arctan \Xi(x, t), & x-X(t)>0,\end{cases}
$$

where

$$
\Xi(x, t):=\sqrt{2 \lambda+1}\left\{\sinh \sqrt{\frac{2 \lambda+1}{1-u^{2}(t)}}[x-X(t)]\right\}^{-1},
$$

and $X(t)=x_{0}+u t$, being $u$ the constant velocity of the soliton. Notice that for positive and negative values of $\lambda$ the potential becomes broader and narrow, respectively (see Fig. 1). As a consequence, the width of the kink at rest changes depending on the value of $\lambda$ (Fig. 2). However, although the potential can be deformed by changing $\lambda$, no local extrema are found, since $|\lambda|<1 / 2$.

We now assume that an approximate solution of Eq. (1) is given by Eq. (2) where the center of the kink $X(t)$ and its velocity $u(t)$ are unknown functions of time, related to each other by the following expression

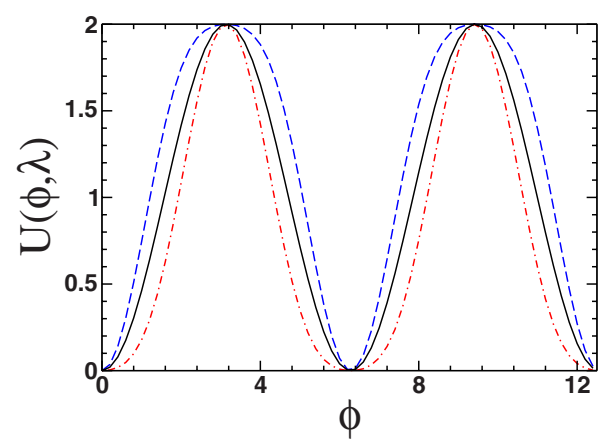

FIG. 1. (Color online). The potential $U(\phi, \lambda)$ of the double sineGordon Eq. (1) for three different values of $\lambda: \lambda=0$ (solid line), $\lambda$ $=0.4$ (dashed blue line), and $\lambda=-0.4$ (dot-dashed red line).

$$
X(t)=x_{0}+\int_{0}^{t} u(\tau) d \tau
$$

Therefore, the ansatz Eq. (2) and (3) proposed in [24] takes into account that small perturbations could affect the dynamics of the kink center, such that its velocity is no longer constant. Indeed, from Eqs. (1) and (2) by using the time derivative of the momentum $P(t) \equiv-\int_{-\infty}^{+\infty} d x \phi_{x} \phi_{t}$, one obtains the following evolution equations for the variables $X(t)$ and $u(t)$

$$
\begin{gathered}
\frac{d u}{d t}=-\frac{q}{M_{0}(\lambda)} f(t)\left(1-u^{2}\right)^{3 / 2}-\beta u\left(1-u^{2}\right), \\
\frac{d X}{d t}=u(t),
\end{gathered}
$$

where $q=2 \pi$ represents the topological charge and
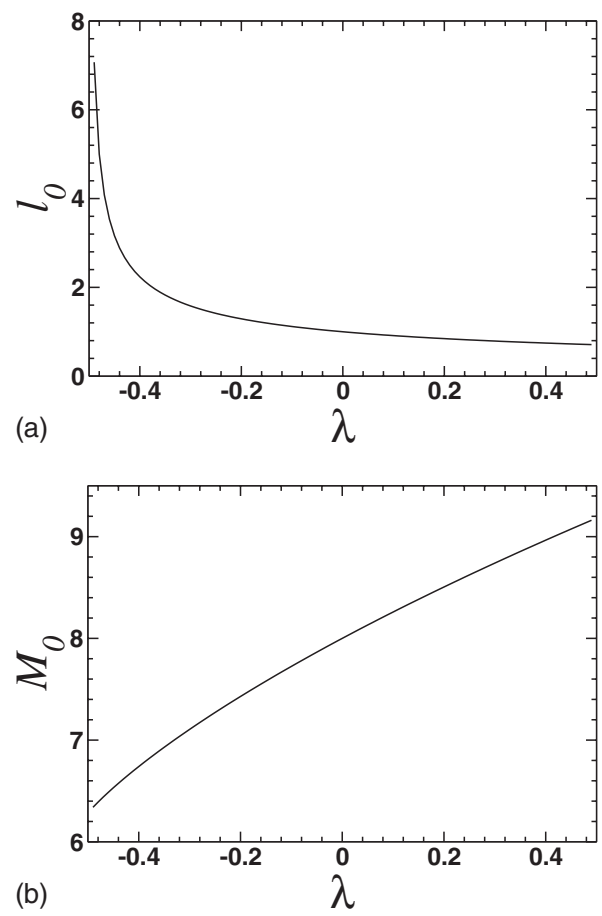

FIG. 2. $l_{0}$ and $M_{0}$ versus $\lambda$. 


$$
M_{0}(\lambda)=4\left(\sqrt{2 \lambda+1}+\frac{1}{\sqrt{2 \lambda}} \arctan h \sqrt{\frac{2 \lambda}{2 \lambda+1}}\right),
$$

is the mass of the kink at rest, which depends on $\lambda$. Increasing the value of $\lambda$ from -0.49 to 0.49 , the mass of the kink is increased. This means that in the point-particle representation the kink is lighter or heavier when $\lambda$ approaches $-1 / 2$ or $1 / 2$, respectively (see Fig. 2). For $\lambda=0, M_{0}=8$ and then Eq. (4) agrees with Eq. (3) of [28], with the Eq. (11) of [11] and with the Eqs. (4.8a) and (4.8b) of [23], where the dynamics of the damped sine-Gordon soliton under the action of an ac force, a biharmonic force, and a constant force was studied, respectively.

In order to solve Eq. (4), notice that the introduction of the variable

$$
P(t)=\frac{M_{0}(\lambda) u(t)}{\sqrt{1-u^{2}(t)}},
$$

transforms Eq. (4) into a linear equation for the momentum

$$
\frac{d P}{d t}=-\beta P-q f(t),
$$

whose solution after $t \gg 1 / \beta$ is given by

$$
P(t)=-\sqrt{\epsilon}\left[a_{1} \sin \left(\omega t+\theta_{1}-\chi_{1}\right)+a_{2} \sin \left(2 \omega t+\theta_{2}-\chi_{2}\right)\right],
$$

where we have introduced $\epsilon \equiv \min \left(\epsilon_{1}, \epsilon_{2}\right)$ as a rescaling parameter [15], and

$$
\begin{gathered}
\chi_{1}=\arctan \left(\frac{\omega}{\beta}\right), \quad \chi_{2}=\arctan \left(\frac{2 \omega}{\beta}\right), \\
a_{1}=\frac{q}{\sqrt{\beta^{2}+\omega^{2}}} \frac{\epsilon_{1}}{\sqrt{\epsilon}}, \quad \text { and } \quad a_{2}=\frac{q}{\sqrt{\beta^{2}+4 \omega^{2}}} \frac{\epsilon_{2}}{\sqrt{\epsilon}} .
\end{gathered}
$$

From Eq. (5) one obtains

$$
\begin{aligned}
u(t)= & \frac{P(t)}{M_{0}(\lambda) \sqrt{1+P^{2} / M_{0}^{2}(\lambda)}}=\frac{P(t)}{M_{0}(\lambda)}-\frac{P^{3}(t)}{2 M_{0}^{3}(\lambda)}+\frac{3}{8} \frac{P^{5}(t)}{M_{0}^{5}(\lambda)} \\
& +\cdots
\end{aligned}
$$

The leading term reads

$$
\langle\dot{X}(t)\rangle=\langle u(t)\rangle=-\frac{1}{2 M_{0}^{3}(\lambda)}\left\langle P^{3}(t)\right\rangle,
$$

where the dot denotes the time derivative and hence,

$$
\begin{aligned}
\langle V\rangle \equiv & \langle\dot{X}(t)\rangle=\frac{3 q^{3}}{8 M_{0}^{3}(\lambda)} \frac{\epsilon_{1}^{2} \epsilon_{2}}{\left(\beta^{2}+\omega^{2}\right) \sqrt{\beta^{2}+4 \omega^{2}}} \\
& \times \sin \left(2 \theta_{1}-\theta_{2}-2 \chi_{1}+\chi_{2}\right) .
\end{aligned}
$$

For $\lambda=0$ this equation agrees with the average ratchet velocity obtained in [11] for the damped and driven sine-Gordon equation. From Eq. (8), one can expect a sinusoidal behavior in $\theta=2 \theta_{1}-\theta_{2}$, a linear behavior in $\epsilon_{2}$ and a quadratic dependence on $\epsilon_{1}$. These features can also be obtained by using symmetry considerations and physical properties of the ratchet effect induced by a biharmonic force [29]. Notice that, since $\langle\dot{X}(t)\rangle \sim 1 / M_{0}^{3}(\lambda)$, a decrease in the ratchet velocity is expected by changing $\lambda$ from -0.49 to 0.49 , i.e. when the internal mode appears for positive values of $\lambda$, the kink mobility is reduced due to the increase in the rest mass of the dsG kink. However, similar to the sine-Gordon case, the expression (8) agrees only qualitatively with the direct numerical simulation of Eq. (1).

\section{B. Second ansatz: two collective coordinates}

In our second approach we present two variables, namely the center of the kink, $X(t)$, and its width, $l(t)$, through Rice's ansatz [26], which amounts to specifying the kink solution as

$$
\phi_{2}(x, t)= \begin{cases}-2 \arctan \Phi(x, t), & x-X(t) \leq 0, \\ 2 \pi-2 \arctan \Phi(x, t), & x-X(t)>0 .\end{cases}
$$

where

$$
\Phi(x, t)=\sqrt{2 \lambda+1}\left(\sinh \frac{x-X(t)}{l(t)}\right)^{-1} .
$$

This ansatz is more general than the one assumed in the previous subsection because $l(t)$ is independent of $X(t)$. Using Eq. (9), and the time variations in momentum and energy

$$
H=\int_{-\infty}^{+\infty}\left(\frac{\phi_{t}^{2}}{2}+\frac{\phi_{x}^{2}}{2}+(1-\cos \phi)+\frac{\lambda}{2}(1-\cos 2 \phi)\right) d x,
$$

it can be shown (in a similar fashion as in [16]) that the momentum still satisfies Eq. (6) and the kink width fulfils the following nonlinear ordinary differential equation

$$
i^{2}-2 \ddot{l}-2 \beta l i=\Omega^{2} l^{2}\left[1+\frac{P^{2}}{M_{0}^{2}(\lambda)}\right]-\frac{1}{\alpha(\lambda)},
$$

where

$$
\begin{gathered}
P(t)=\frac{M_{0}(\lambda) l_{0}(\lambda) \dot{X}}{l(t)}, \quad l_{0}(\lambda)=\frac{1}{\sqrt{2 \lambda+1}}, \\
\Omega(\lambda)=\frac{1}{\sqrt{\alpha(\lambda)} l_{0}(\lambda)}, \quad \alpha(\lambda)=\frac{I_{3}}{I_{1}},
\end{gathered}
$$

being

$$
\begin{gathered}
I_{1}=\int_{-\infty}^{+\infty} \frac{\cosh ^{2} z}{\left(2 \lambda+\cosh ^{2} z\right)^{2}} d z=\frac{M_{0}(\lambda)}{4(2 \lambda+1)^{3 / 2}}, \\
I_{3}=\int_{-\infty}^{+\infty} \frac{z^{2} \cosh ^{2} z}{\left(2 \lambda+\cosh ^{2} z\right)^{2}} d z .
\end{gathered}
$$

From now on, the notation $\Omega=\Omega(\lambda)$ for the Rice frequency, $l_{0}=l_{0}(\lambda)$ and $M_{0}=M_{0}(\lambda)$ for the width and the mass of the kink at rest, respectively (see Fig. 2), and $\alpha=\alpha(\lambda)$ is used. We would like to remark that the frequency $\Omega$ of $l(t)$ for positive values of $\lambda$ practically coincides with the frequency of the localized internal mode (see upper panel Fig. 3 ), although for some positive values close to zero $\Omega$ pen- 
etrates in the phonon band. This behavior is even clearer for negative values of $\lambda$, where the Rice ansatz predicts an oscillations of the kink width with $\Omega$, while from the linear spectrum around a static kink it is known that the localized IM, which appears for $\lambda>0$, becomes a quasilocalized phonon mode when $\lambda<0$ (see lower panel Fig. 3), so $\Omega$ could be interpreted as an effective frequency of the lower phonon modes.

We now analyze Eq. (10) where the solution for $P(t)$ is represented by Eqs. (7) and (8). Due to the term $P^{2}$ on the right-hand side of Eq. (10), it is clear that $l(t)$ is indirectly driven by the harmonic forces with frequencies $\omega$ and $2 \omega$. To solve (approximately) this equation, we can expand $l(t)$ in powers of $\epsilon$ around the unperturbed kink width $l_{0}$

$$
l(t)=l_{0}+\epsilon l_{1}(t)+\epsilon^{2} l_{2}(t)+\ldots
$$

Substituting Eq. (11) into Eq. (10) we find a hierarchy of equations for different orders of powers in $\epsilon$. The first one is given by

$$
\begin{aligned}
\ddot{l}_{1}(t)+\beta i_{1}(t)+\Omega^{2} l_{1}(t)= & A_{1}+A_{2} \cos \left(2 \omega t+2 \theta_{1}-2 \chi_{1}\right) \\
& +A_{3} \cos \left(4 \omega t+2 \theta_{2}-2 \chi_{2}\right) \\
& +A_{4}\left\{\cos \left[\omega t+\theta_{2}-\theta_{1}-\left(\chi_{2}-\chi_{1}\right)\right]\right. \\
& \left.-\cos \left[3 \omega t+\theta_{1}+\theta_{2}-\left(\chi_{2}+\chi_{1}\right)\right]\right\}
\end{aligned}
$$

where

$$
\begin{aligned}
& A_{1}=-A_{2}-A_{3}, \quad A_{2}=\frac{\Omega a_{1}^{2}}{4 \sqrt{\alpha} M_{0}^{2}}, \\
& A_{3}=\frac{\Omega a_{2}^{2}}{4 \sqrt{\alpha} M_{0}^{2}}, \quad A_{4}=-\frac{\Omega a_{1} a_{2}}{2 \sqrt{\alpha} M_{0}^{2}} .
\end{aligned}
$$

Notice on the right-hand side of Eq. (12) the presence of harmonics with frequencies $\omega, 2 \omega, 3 \omega$, and $4 \omega$. Consequently, all these frequencies appear in the expression for $l_{1}(t)$ and they remain for $t \gg 1 / \beta$, i.e., asymptotically we have

$$
\begin{aligned}
l_{1}(t)= & \frac{A_{1}}{\Omega^{2}}+\frac{A_{2} \sin \left(2 \omega t+2 \theta_{1}-2 \chi_{1}+\tilde{\theta}_{2}\right)}{\sqrt{\left(\Omega^{2}-4 \omega^{2}\right)^{2}+4 \beta^{2} \omega^{2}}} \\
& +\frac{A_{3} \sin \left(4 \omega t+2 \theta_{2}-2 \chi_{2}+\tilde{\theta}_{4}\right)}{\sqrt{\left(\Omega^{2}-16 \omega^{2}\right)^{2}+16 \beta^{2} \omega^{2}}} \\
& +\frac{A_{4} \sin \left[\omega t+\theta_{2}-\theta_{1}-\left(\chi_{2}-\chi_{1}\right)+\tilde{\theta}_{1}\right]}{\sqrt{\left(\Omega^{2}-\omega^{2}\right)^{2}+\beta^{2} \omega^{2}}} \\
& -\frac{A_{4} \sin \left[3 \omega t+\theta_{1}+\theta_{2}-\left(\chi_{2}+\chi_{1}\right)+\tilde{\theta}_{3}\right]}{\sqrt{\left(\Omega^{2}-4 \omega^{2}\right)^{2}+\beta^{2} 4 \omega^{2}}},
\end{aligned}
$$

where
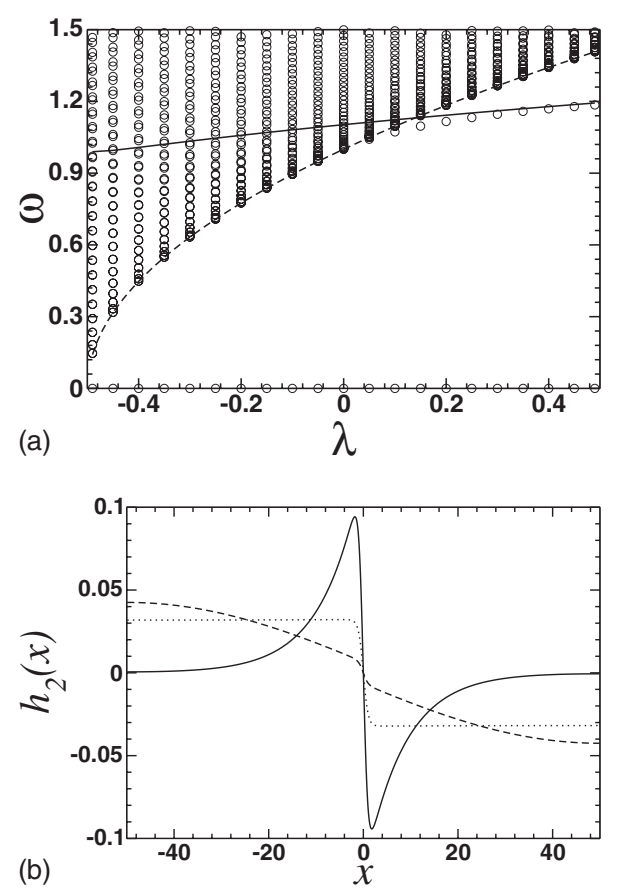

FIG. 3. Upper panel: computed spectrum of the linearized dsG equation versus $\lambda . \omega=0$ is the Goldstone mode. Circles below the dashed line, $\omega_{p h}=\sqrt{2 \lambda+1}$, correspond to the internal mode. The solid line is the curve $\Omega(\lambda)$. The other open circles represent the lowest phonon modes. Lower panel: it is shown the second computed eigenfunction related to the linear spectrum of the dsG equation. $h_{2}(x)$ (solid line) corresponds to the localized IM for $\lambda=0.05$, while $\lambda=0$ (dotted line) and $\lambda=-0.05$ (dashed line) are the first quasilocalized phonon modes.

$$
\tilde{\theta}_{m}=\arctan \left(\frac{\Omega^{2}-m^{2} \omega^{2}}{m \beta \omega}\right) .
$$

Similarly, one can find the harmonics corresponding to the second and third-order corrections $l_{2}(t)$ and $l_{3}(t)$. We can now calculate the velocity averaged over one period $T$. To this end, we use the previously defined expression for the momentum, $P(t)=M_{0} l_{0} \dot{X} / l(t)$. After transients have elapsed and using Eq. (11) the average velocity of the kink can be expressed in terms of the $\mathrm{CC}$ as

$$
\begin{aligned}
\langle\dot{X}(t)\rangle= & \frac{1}{T} \int_{0}^{T} \frac{P(t) l(t)}{M_{0} l_{0}} d t \\
= & \frac{1}{T} \int_{0}^{T} \frac{P(t)\left(l_{0}+\epsilon l_{1}(t)+\epsilon^{2} l_{2}(t)+\ldots\right)}{M_{0} l_{0}} d t=\left\langle\dot{X}_{0}(t)\right\rangle \\
& +\epsilon\left\langle\dot{X}_{1}(t)\right\rangle+\epsilon^{2}\left\langle\dot{X}_{2}(t)\right\rangle+\ldots
\end{aligned}
$$

Therefore, the average velocity can be analytically calculated taking into account the exact solution for the momentum Eq. (6) and the first terms of the expansion for the kink width. At order $O\left(\epsilon^{0}\right),\left\langle\dot{X}_{0}(t)\right\rangle \sim\langle P(t)\rangle=0$ since the average of the momentum is exactly zero as has been shown in [30]. Accordingly, the net motion of the kink can only arise from the contribution of higher order terms; hence, we proceed by 
computing the integral defining $\epsilon\left\langle\dot{X}_{1}(t)\right\rangle$. Inserting Eqs. (6) and (13) in Eq. (14), straightforward calculations show that

$$
\langle V\rangle \equiv \epsilon\left\langle\dot{X}_{1}\right\rangle=-\epsilon_{1}^{2} \epsilon_{2} \Gamma_{1}(\beta, \omega, \lambda) \sin \left[\theta_{2}-2 \theta_{1}+\Phi_{1}(\beta, \omega, \lambda)\right],
$$

where $\Gamma_{1}$ is the amplitude

$$
\Gamma_{1}(\beta, \omega, \lambda)=\frac{q^{3} \Omega^{2}}{8 M_{0}^{3}\left(\beta^{2}+\omega^{2}\right) \sqrt{\beta^{2}+4 \omega^{2}}} \times \sqrt{\frac{4\left(\Omega^{2}-4 \omega^{2}\right)^{2}+\left(\Omega^{2}-\omega^{2}\right)^{2}+4\left(\Omega^{2}-\omega^{2}\right)\left(\Omega^{2}-4 \omega^{2}\right)+9 \beta^{2} \omega^{2}}{\left[\left(\Omega^{2}-\omega^{2}\right)^{2}+\beta^{2} \omega^{2}\right]\left[\left(\Omega^{2}-4 \omega^{2}\right)^{2}+4 \beta^{2} \omega^{2}\right]}},
$$

and $\Phi_{1}$ is the phase

$$
\begin{gathered}
\Phi_{1}(\beta, \omega, \lambda)=2 \chi_{1}-\chi_{2}-\arctan \Gamma, \\
\Gamma=\frac{2 \beta \omega\left[\left(\Omega^{2}-4 \omega^{2}\right)^{2}+3 \beta^{2} \omega^{2}-\left(\Omega^{2}-\omega^{2}\right)^{2}\right]}{2\left(\Omega^{2}-\omega^{2}\right)\left[\left(\Omega^{2}-4 \omega^{2}\right)^{2}+4 \beta^{2} \omega^{2}\right]+\left(\Omega^{2}-4 \omega^{2}\right)\left[\left(\Omega^{2}-\omega^{2}\right)^{2}+\beta^{2} \omega^{2}\right]},
\end{gathered}
$$

which are functions not only of the dissipation coefficient and the frequencies of the ac force, but also of $\lambda$ through $\Omega$ (see the upper panel in Fig. 3 in which one observes that $\Omega(\lambda)$ undergoes a tiny change around one when $\lambda$ is changed). Notice also that for the first ansatz we obtained the same expression (15) [see Eq. (8)] but seemingly with different amplitude and phase. Nevertheless, one can show that, if $\omega \ll \Omega$ and $\beta \ll 1$, then $\Gamma_{1}$ and $\Phi_{1}$ tend to the amplitude and the frequency, respectively, of $\langle\dot{X}(t)\rangle$ given by Eq. (8). It is important to recall that for the $\mathrm{CC}$ approach to be valid, we must require that the kink shape is not very much distorted, and hence that $\epsilon_{i} \ll 1$ and $\omega \ll \omega_{p h}$, where $\omega_{p h}$ is the lower phonon band edge which depends on $\lambda$ (upper panel in Fig. $3)$.

From the above we realize that the approximate expression for the ratchet velocity is not improved by using the Rice ansatz, compared to the ansatz with only one CC. However, the ratchet mechanism can be clarified. Indeed, by writing the equation that is now fullfiled by $X(t)$, in the form

$$
\ddot{X}+\beta \dot{X}-\frac{\dot{X} \dot{l}}{l}=-\frac{q l(t)}{M_{0} l_{0}} f(t),
$$

it is easy to see that the kink center is driven by an effective force, which can not be zero on the average due to the oscillations of $l(t)$. This expression has been obtained in [16], where the authors compared the mobility of sG and $\phi^{4}$ kinks. For sG $q=2 \pi, M_{0}=8$, and $l_{0}=1$, and for $\phi^{4} q=2, M_{0}$ $=2 \sqrt{2} / 3$, and $l_{0}=\sqrt{2}$. Since $l(t)$ oscillates around $l_{0}$, the amplitude of the effective force is modulated by $q / M_{0}$, and hence the $\phi^{4}$ kink exhibits a higher ratchet velocity. However, in this case we cannot conclude anything about the role of the internal mode for the ratchet mobility. In the dsG, for positive values of $\lambda$ an internal mode appears, but while $q$ remains invariant $M_{0}$ increases, and so the velocity decreases instead of increasing. It is interesting to remark that by changing $\lambda$ from 0 to $-0.49, M_{0}$ decreases from 8 to 6.3 and then a higher kink mobility is expected in spite of the nonexistence of an internal mode.

\section{NUMERICAL SOLUTIONS OF THE CC EQUATIONS AND SIMULATIONS OF THE PARTIAL DIFFERENTIAL EQUATION}

We compare the analytical results obtained in the previous section with those of numerical simulations of Eq. (1) and numerical solutions of Eq. (4), obtained by using the 1-CC theory, and Eqs. (6) and (10) obtained via the 2-CC approach. The numerical solutions and simulations were performed by a fourth-order Runge-Kutta scheme with space and time steps $\Delta x=0.1, \Delta t=0.01$, respectively, in the finite length domain $x \in[-50,50]$ using as initial condition a static dsG kink at $x=0$.

First, in Fig. 4 the sinusoidal dependence on the phase of the second harmonic, predicted by both $\mathrm{CC}$ theories, is verified. Moreover, we observe that the maximum of the ratchet velocity decreases when $\lambda$ goes from 0 to 0.49 , while if $\lambda$ approaches $-1 / 2$ from $0,\langle V\rangle$ is increased. From Eqs. (15)-(17) it is expected that the zeros of $\langle V\rangle$ depend on $\lambda$, however this dependence is so weak that it is not visible on the scale of Fig. 4, where for this set of parameters $\Phi_{1}$ $\approx 2 \chi_{1}-\chi_{2}=0.88848$.

In Fig. 5 we compare the simulations of the partial differential equation (PDE) with the numerical solutions of the $\mathrm{CC}$ equations and with the formulas (8) and (15) predicted for the ratchet velocity by using the 1-CC and 2-CC theories, respectively. For the negative value $\lambda=-0.45$ (see upper panel) the numerical solutions of the 1-CC and 2-CC equations and the corresponding analytical results for the ratchet velocity show only a qualitative agreement with the simulation results. However, for the positive value $\lambda=0.45$ (see lower panel) one observes that the numerical solutions of the $\mathrm{CC}$ equations and the simulation results are practically identical.

Another interesting feature from Fig. 5 is that the blue circles agree with the dot-dashed ones, while the red squares agree with the dashed ones. This means that the 2-CC theory used in the previous section does not improve the results obtained by the 1-CC theory, in contrast with other soliton ratchets [9]. In this regard, it has recently been found for the damped and driven $\mathrm{sG}$ equation with additive inhomogene- 

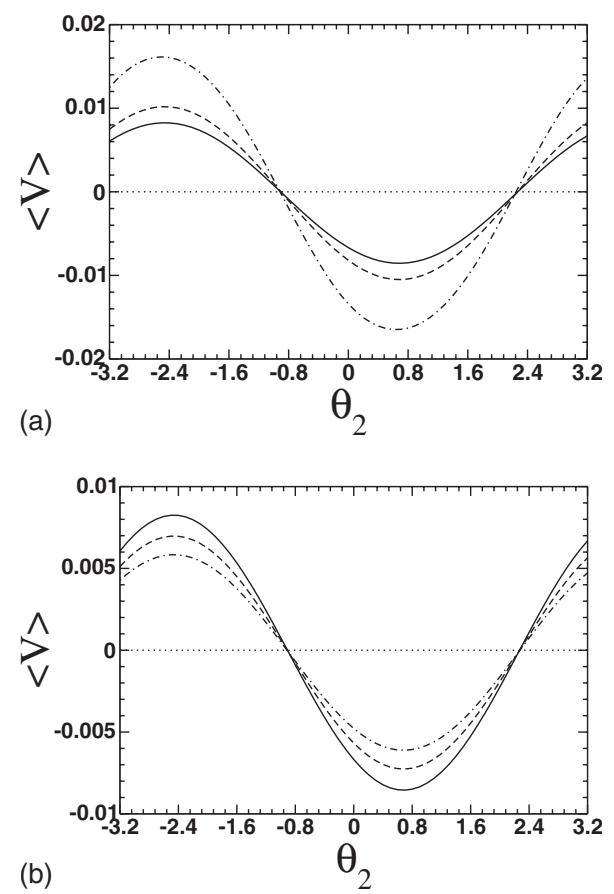

FIG. 4. Simulations for Eq. (1). Average velocity versus $\theta_{2}$ for different values of $\lambda$. Upper panel: $\lambda=0$ (solid line), $\lambda=-0.2$ (dashed line), and $\lambda=-0.45$ (dot-dashed line). Lower panel: $\lambda=0$ (solid line), $\lambda=0.2$ (dashed line), and $\lambda=0.45$ (dot-dashed line). Parameters of the simulations: $\beta=0.05 ; \omega=0.1 ; \epsilon_{1}=2 / 30 ; \quad \epsilon_{2}$ $=1 / 30 ; \theta_{1}=0$.

ities [31] that the ratchet velocity is only improved when the oscillations of the background are taken into account.

Similar results are obtained for other values of $\lambda$, i.e. for fixed $\lambda \in[-0.49 ; 0.49]$ a sinusoidal dependence of $\langle V\rangle$ on $\theta_{2}$ is found. Then, if we compute the maximum of this function, and plot this value versus $\lambda$ (see Fig. 6), we realize, first, that the agreement between simulations and numerical solutions of the CC equations is better for positive values of $\lambda$; and, second, that the ratchet mobility surprisingly does not increase when the internal mode appears (i.e. when $\lambda>0$ ). Moreover, the average velocity rises up to 1.76 times (the prediction from the $\mathrm{CC}$ theories is 2 ) when $\lambda$ goes from 0 to -0.49 .

In order to improve our analytical results for negative $\lambda$ we use a 3-CC theory developed in [31] for the perturbed $\mathrm{sG}$ equation. So, first we assume that our ansatz is $\phi_{3}(x, t)$ $=\phi_{2}(x, t)+\varphi(t)$, where $\phi_{2}(x, t)$ is the Rice ansatz given by Eq. (9) and $\varphi(t)$ represents the kink offset (up-and-down oscillations of the whole kink). Second, we can apply the generalized traveling wave ansatz (GTWA) (see [31] for details) and finally we obtain that $X(t)$ and $\varphi(t)$ satisfy

$$
\begin{gathered}
\ddot{X}+\beta \dot{X}-\frac{\dot{X} \dot{l}}{l}=-\frac{q l(t)}{M_{0} l_{0}} \sin (\varphi), \\
\ddot{\varphi}+\beta \dot{\varphi}+\sin (\varphi)=f(t),
\end{gathered}
$$

where $l(t)$ fulfills the Eq. (10). The numerical solutions of the 3-CC equations yield a substantial improvement compared to
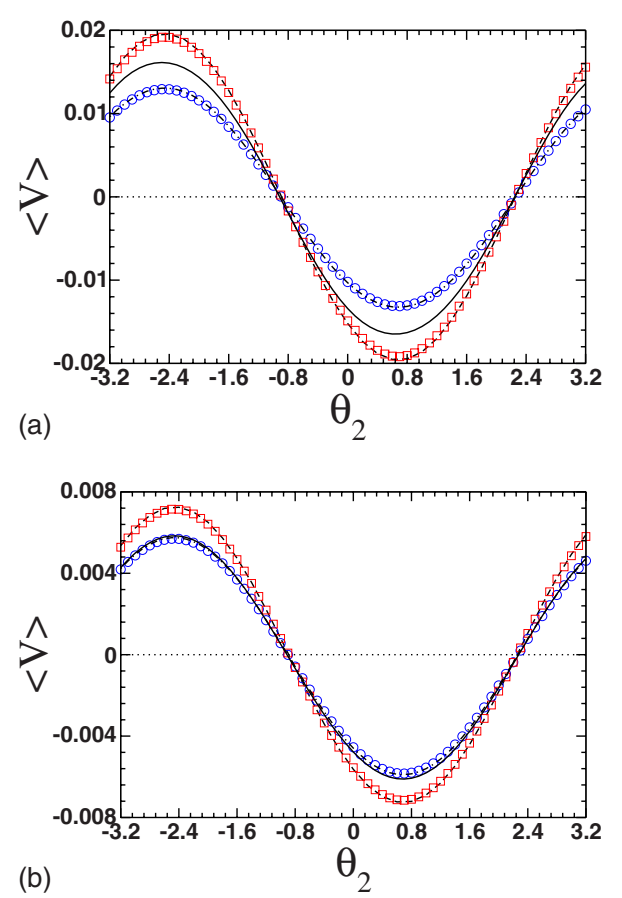

FIG. 5. (Color online). Average velocity versus $\theta_{2}$. Simulations of Eq. (1) (black solid lines), numerical solutions of the 1-CC and 2-CC equations (blue circles and dot-dashed lines) and approximate expressions (8) and (15) for $\langle V\rangle$ (red squares and dashed lines). Upper panel: $\lambda=-0.45$. Lower panel: $\lambda=0.45$. Other parameters as in Fig. 4.

the 2-CC theory (Fig. 6). However, when $\lambda$ approaches $-1 / 2$ there is still a difference between simulations and numerical solutions.

For positive $\lambda$ the rest width $l_{0}$ is in the order of one and depends only weakly on $\lambda$ (Fig. 2) and therefore the 2-CC theory, which includes the width $l(t)$ as a second collective variable, works well here. However, for negative $\lambda$ and particularly for $\lambda$ approaching the value -0.5 , the rest width increases strongly with $\lambda$. Here the kink really behaves like an extended soft object and therefore the 2-CC theory agrees only qualitatively with the simulations.

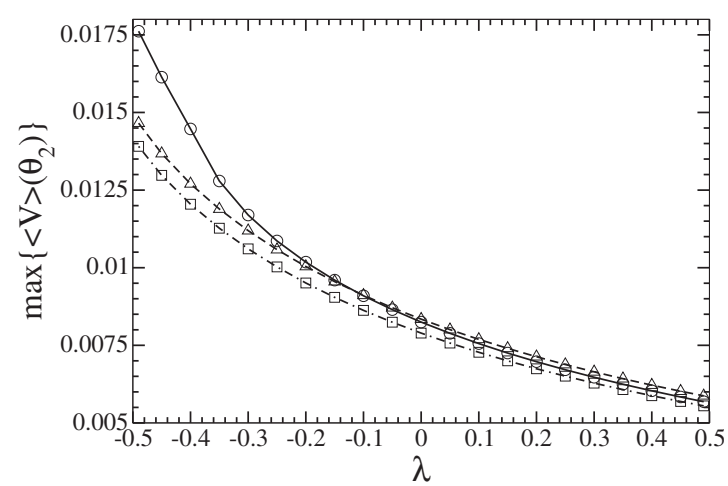

FIG. 6. Maximum of average velocity versus $\lambda$. Simulations of the PDE (circles joined by a solid line) and numerical solutions of the 2-CC equations (squares joined by a dot-dashed line) and the 3-CC equations (triangles joined by a dashed line). Parameters as in Figs. 4 and 5. 
Moreover, for positive $\lambda$ the kink profile exhibits an upand-down oscillation of the whole kink while it is moving. For this reason the inclusion in the third $\mathrm{CC}$, which represents the kink offset, improves the results obtained from the 2-CC theory. However, for negative values of $\lambda$ the offset does not only depend on time, but also on space. This means that the phonons, which are not taken into account by our 2and 3-CC theories, are excited by the biharmonic force. Indeed, notice that although $f(t)$ oscillates with $\omega=0.1$ and $2 \omega=0.2$, in the spectrum of the first-order correction of the kink width [Eq. (13)] appear not only these two frequencies but also the frequencies $3 \omega=0.3$ and $4 \omega=0.4$, which are either very close to the lower phonon band edge or even penetrate into the phonon band when $\lambda$ approaches -0.5 (see Fig. 3).

\section{CONCLUSIONS}

In this work we have studied the influence of the internal mode (IM) on the kink ratchet mobility in the damped double sine-Gordon ( $\mathrm{dsG}$ ) equation driven by a biharmonic force. In this system a parameter $\lambda(|\lambda|<1 / 2)$ controls the existence and the frequency of the IM, which appears only for $\lambda>0$. It turns out that by using a 1-CC theory with the kink center $X(t)$ as a collective coordinate, the ratchet velocity $\langle V\rangle \sim 1 / M_{0}^{3}(\lambda)$ [see Eq. (8)], where $M_{0}(\lambda)$ is the rest mass of the kink, which grows from 6.3 to 9.2 when $\lambda$ changes from -0.49 to +0.49 , i.e., when the IM appears for positive values of $\lambda$, the kink mobility is reduced due to the inertial effect on the dsG kink. In order to investigate the influence of the IM on the ratchet velocity we have used the Rice ansatz, which takes into account the kink width $l(t)$ as an additional CC, independent of $X(t)$. Within this approach we have obtained an approximate expression for $\langle V\rangle[$ see Eq. (15)], which shows the influence of the IM through $\Omega(\lambda)$ (the Rice frequency in the dsG). However, for $\omega \ll \omega_{p h}$ and $\beta$ $\ll 1$ this effect is practically negligible because of the tiny change of $\Omega$ when $\lambda$ changes (see Fig. 3). For the set of parameters considered in Fig. 4 , the relative change in the amplitude $\Gamma_{1}$ in Eq. (15) is $\Gamma_{1}(0.05,0.1$, $-0.49) / \Gamma_{1}(0.05,0.1,+0.49)=1.0066$.

A direct comparison between the simulations of the PDE and numerical solutions of the 1-CC Eq. (4) and the 2-CC Eqs. (6) and (10) shows that the generic features of the soliton ratchets observed and studied in other nonlinear driven Klein-Gordon equations driven by the same biharmonic force $f(t)[16]$ also appear in the dsG. Indeed, the sinusoidal dependence of the average velocity on the phase of the second harmonic is verified, and a complex behavior of $\langle V\rangle$ versus the damping coefficient has been found. It has been obtained a better agreement between the simulations and numerical solutions when the IM appears for positive values of $\lambda$, even using only 1-CC theory. In addition, it is found that in contrast with other soliton ratchets [9] the 2-CC ansatz does not improve the ratchet velocity. On the other hand, for negative values of $\lambda$ we can improve the CC results by including one more collective coordinate, the kink offset $\varphi(t)$. Nevertheless, when $\lambda$ approaches $-1 / 2$ a difference of $14 \%$ in the maximum of the average velocity obtained by means of the simulations of Eq. (1) and numerical solutions of 3-CCs equations is observed.

In summary, although in the dsG the IM appears for positive $\lambda$ allowing the kink width to oscillate with the IM frequency, simultaneously the kink becomes heavier, and so this strong inertial effect reduces the ratchet velocity. When the IM disappears and becomes a quasilocalized phonon mode for negative $\lambda$, the kink mobility is enhanced because the kink is lighter but more distorted. Our study, which includes positive and negative values of $\lambda$, will be important in the context of magnetic systems, where, depending on the values of anisotropies and applied fields, all values of $\lambda$ are possible [19]. We expect that the ratchet mobility of the dsG model studied here has implications on the conductivity properties of driven nonlinear chains modeled not only by the dsG, but also by other nonlinear Klein-Gordon potentials such as for instance the Peyrard-Remoissenet potential [22], where there is also a parameter that controls the existence of the IM and the kink mass in the same way. Furthermore, a similar influence of internal mode on the gating ratchet mobility is expected when an additive and a parametric driving are used instead of a biharmonic one in order to induce a ratchet effect [32].

\section{ACKNOWLEDGMENTS}

We acknowledge financial support by the Ministerio de Educación y Ciencia (MEC, Spain) through Grants No. MTM2006-13000-C03-01 (RAN) and No. FIS2008-02380/ FIS (NRQ), and by the Junta de Andalucía under the projects "Estancias de excelencia 2008/2" (NRQ), No. FQM262 (RAN), No. FQM207 (NRQ), No. 00481 and No. P06-FQM01735 (RAN,NRQ). F.M. thanks the Instituto de Investigación de la Universidad de Sevilla (IMUS) for financial support and kind hospitality.

\section{APPENDIX: LINEAR SPECTRUM AROUND THE STATIC KINK SOLUTION IN THE DSG}

The stability of the kink solution in the dsG equation was numerically studied in [19] and commented in [33] (see also the references therein). Notice, however that the factors in the potential that was used in these references differ from ours. These factors can be changed by scaling the time and space variables, but in any case it is too difficult to compare the Rice frequency obtained by the CC approach developed in the previous section with the numerical results in [19] for the internal mode of the $\mathrm{dsG}$ equation. For this reason we perform here the linear stability analysis for the pure dsG equation. We follow a standard procedure developed in [34] for the $\mathrm{sG}$ equation. First one takes in Eq. (1) $\beta=0$ and $f(t)=0$ in order to obtain the unperturbed dsG equation, which is Lorentz invariant. Then, without loss of generality, this equation is linearized around its exact static kink solution, $\phi_{1}(x)$ (take in Eq. (2) $u=0$ and $X(t)=0$ ), i.e., it is assumed that the solution of the unperturbed $\mathrm{dsG}$ is $\phi_{1}(x)$ $+\psi(x, t)$, where $|\psi(x, t)| \ll 1[34]$. This leads us to the following linear problem for $\psi(x, t)$

$$
\psi_{t t}-\psi_{x x}+\left[\cos \left(\phi_{1}\right)+2 \lambda \cos \left(2 \phi_{1}\right)\right] \psi=0,
$$




$$
-\infty<x<+\infty, \quad \psi_{x}( \pm \infty)=0,
$$

whose solution can be expressed as

$$
\psi(x, t)=\exp (i \omega t) h(x), \quad h_{x}( \pm \infty)=0 .
$$

Second, by substituting Eq. (19) into Eq. (18) we finally obtain that $h(x)$ satisfies

$$
\begin{gathered}
h_{x x}+\left[\omega^{2}-\cos \left(\phi_{1}\right)-2 \lambda \cos \left(2 \phi_{1}\right)\right] h=0, \\
-\infty<x<+\infty, \quad h_{x}( \pm \infty)=0 .
\end{gathered}
$$

For the $\mathrm{sG}$ equation, i.e when $\lambda=0$, this eigenvalue problem can be exactly solved [34]. When $\lambda \neq 0$, this equation can be numerically solved for any finite length of the system.

By using the QR algorithm we have solved numerically Eq. (20), fixing $L=100, \Delta x=0.1$, and taking different values of $\lambda$ between -0.5 and 0.5 . We have obtained that for 0 $<\lambda \leq 0.49$, the Eq. (20) has an internal mode with a frequency $\Omega_{i}$ below the spectrum of the phonon band (Fig. 3). For $\lambda=0$ we have not found any internal mode as is expected for integrable systems [35], however for negative values of $\lambda$ $(-0.49 \leq \lambda<0)$, we have not found any internal mode, although the system is not integrable (see Fig. 3). This feature of the linear spectrum around a kink solution is also observed in other nonintegrable Klein-Gordon systems, as for instance the PR model [22].
[1] P. Reimann, Phys. Rep. 361, 57 (2002).

[2] P. Hänggi and R. Bartussek, Nonlinear Physics of Complex Systems-Current Status and Future Trends, Lecture notes in Physics Vol. 746, edited by J. Parisi et al. (Springer, Berlin, 1996), p. 476.

[3] R. D. Astumian and P. Hänggi, Phys. Today 55(11), 33 (2002).

[4] P. Hänggi, F. Marchesoni, and F. Nori, Ann. Phys. 14, 51 (2005).

[5] S. Cilla, F. Falo, and L. M. Floría, Phys. Rev. E 63, 031110 (2001).

[6] A. R. Bishop, J. A. Krumhansl, and S. E. Trullinger, Physica D 1, 1 (1980).

[7] F. Marchesoni, Phys. Rev. Lett. 77, 2364 (1996).

[8] M. Salerno and N. R. Quintero, Phys. Rev. E 65, 025602(R) (2002).

[9] L. Morales-Molina, F. G. Mertens, and A. Sánchez, Eur. Phys. J. B 37, 79 (2004); F. G. Mertens, L. Morales-Molina, A. R. Bishop, A. Sánchez, and P. Müller, Phys. Rev. E 74, 066602 (2006).

[10] N. R. Quintero, B. Sánchez-Rey, and M. Salerno, Phys. Rev. E 72, 016610 (2005).

[11] M. Salerno and Y. Zolotaryuk, Phys. Rev. E 65, 056603 (2002).

[12] A. V. Ustinov, C. Coqui, A. Kemp, Y. Zolotaryuk, and M. Salerno, Phys. Rev. Lett. 93, 087001 (2004).

[13] S. Ooi, S. Savel'ev, M. B. Gaifullin, T. Mochiku, K. Hirata, and F. Nori, Phys. Rev. Lett. 99, 207003 (2007).

[14] I. Zapata, R. Bartussek, F. Sols, and P. Hänggi, Phys. Rev. Lett. 77, 2292 (1996); E. Trías, J. J. Mazo, F. Falo, and T. P. Orlando, Phys. Rev. E 61, 2257 (2000); M. Beck, E. Goldobin, M. Neuhaus, M. Siegel, R. Kleiner, D. Koelle, Phys. Rev. Lett. 95, 090603 (2005).

[15] L. Morales-Molina, N. R. Quintero, F. G. Mertens, and A. Sánchez, Phys. Rev. Lett. 91, 234102 (2003).

[16] L. Morales-Molina, N. R. Quintero, A. Sánchez, and F. G. Mertens, Chaos 16, 013117 (2006).
[17] N. R. Quintero, A. Sánchez, and F. G. Mertens, Phys. Rev. E 62, R60 (2000); 73, 068602 (2006).

[18] N. R. Quintero and P. G. Kevrekidis, Phys. Rev. E 64, 056608 (2001).

[19] D. K. Campbell, M. Peyrard, and P. Sodano, Physica D 19, 165 (1986).

[20] S. Pnevmatikos, Phys. Rev. Lett. 60, 1534 (1988).

[21] Y. S. Kivshar, D. E. Pelinovsky, T. Cretegny, and M. Peyrard, Phys. Rev. Lett. 80, 5032 (1998).

[22] M. Remoissenet and M. Peyrard, J. Phys. C 14, L481 (1981); M. Peyrard and M. Remoissenet, Phys. Rev. B 26, 2886 (1982); M. Peyrard and D. K. Campbell, Physica D 9, 33 (1983).

[23] D. W. McLaughlin and A. C. Scott, Phys. Rev. A 18, 1652 (1978).

[24] C. A. Popov, Wave Motion 42, 309 (2005).

[25] N. R. Quintero, A. Sánchez, and F. G. Mertens, Phys. Rev. E 62, 5695 (2000).

[26] M. J. Rice and E. J. Mele, Solid State Commun. 35, 487 (1980); M. J. Rice, Phys. Rev. B 28, 3587 (1983).

[27] A. Sánchez and A. R. Bishop, SIAM Rev. 40, 579 (1998).

[28] N. R. Quintero and A. Sánchez, Phys. Lett. A 247, 161 (1998).

[29] R. Chacón and N. R. Quintero, Biosystems 88, 308 (2007).

[30] N. R. Quintero, B. Sánchez-Rey, and J. Casado-Pascual, Phys. Rev. E 71, 058601 (2005).

[31] V. Stehr, P. Müller, F. G. Mertens, and A. Bishop, Phys. Rev. E 79, 036601 (2009).

[32] E. Zamora-Sillero, N. R. Quintero, and F. G. Mertens, Phys. Rev. E 74, 046607 (2006).

[33] J. A. Holyst and H. Benner, Phys. Rev. E 52, 4583 (1995).

[34] M. B. Fogel, S. E. Trullinger, A. R. Bishop, and J. A. Krumhansl, Phys. Rev. B 15, 1578 (1977).

[35] O. V. Charkina and M. M. Bogdan, in Symmetry, Integrability and Geometry: Methods and Applications, Vol 2, (2006) Paper No. 047. 